\title{
Drag on pairs of square section obstacles in free-surface flows
}

\author{
Francis H. Robertson ${ }^{*}$ and Gregory F. Lane-Serff ${ }^{\dagger}$ \\ School of Mechanical, Aerospace and Civil Engineering, The University of Manchester, \\ Manchester M13 9PL, United Kingdom
}

(Received 21 August 2017; revised manuscript received 12 September 2018; published 18 December 2018)

\begin{abstract}
The drag on pairs of square obstacles (of side $D$ ) in open channel flow is measured in experiments using a laboratory flume. The depth is uniform across the obstacles and the conditions in the flume are subcritical, with Froude number $\mathrm{Fr}<0.59$, Reynolds number $\mathrm{Re}=4800$ to 21900 , and turbulence intensity $I_{T} \sim 8$ to $10 \%$. The drag coefficient for an isolated square obstacle is found to be $C_{D}=2.11$, in agreement with previous studies, and independent of $\mathrm{Re}$ (for the range covered here). The root-mean-square variation in the drag coefficient for the single obstacle decreased monotonically with Re, defined in terms of hydraulic radius, and approached 0.241 at high $\mathrm{Re}$ in agreement with previous research. For two obstacles, standard tandem and side-by-side arrangements are studied first, followed by a full range of relative positions covering $-5 \leqslant s_{x} / D \leqslant 20$ in the downstream direction and $0 \leqslant s_{y} / D \leqslant 7.0$ in the cross-stream direction. The lowest drag coefficients are observed when the downstream obstacle is shielded directly behind the upstream obstacle (tandem arrangement) when negative drag coefficients are found. The largest drag coefficients are observed for nearly side-by-side arrangements, with the peak values found to be for the slightly upstream obstacle of a pair $\left(s_{x} / D=-1\right)$. The blockage ratio $(D / B$, the relative size of the obstacle compared to the channel width) is found to be an important factor. For $D / B=12.7 \%$ the largest drag coefficient is $C_{D}=3.82$, while for $D / B=6.3 \%$ the largest value is $C_{D}=2.85$. For tandem obstacles, the drag on one obstacle can largely be accounted for by the change in flow speed induced by the other obstacle, except at small separations $\left(\left|s_{x} / D\right|<3\right)$. The results will be useful in any applications where the force on multiple obstacles is required, such as the design of marine or riverine structures, or flood flows past buildings and vegetation.
\end{abstract}

DOI: 10.1103/PhysRevFluids.3.123802

\section{INTRODUCTION}

Bluff obstacles are found in a range of natural and engineered flows. For example, square cross-section obstacles are found as supports for river and coastal structures, buildings experiencing floods, and in heat exchangers [1-4]. Emergent aquatic vegetation is also often idealized as arrays of rigid obstacles, typically with a circular cross section (e.g., [5-7]), though it is argued here that square obstacles can be a more appropriate model for real vegetation under typical field conditions

\footnotetext{
*Present address: School of Engineering, The University of Birmingham, Edgbaston, Birmingham B15 2TT, United Kingdom.

†g.f.lane-serff@manchester.ac.uk

Published by the American Physical Society under the terms of the Creative Commons Attribution 4.0 International license. Further distribution of this work must maintain attribution to the author(s) and the published article's title, journal citation, and DOI.
} 
in terms of the magnitude of fluid drag and its relatively small variation with Reynolds number. We will focus here on free surface subcritical flow, with the Froude number $\mathrm{Fr}<1$, and Froude number given by

$$
\operatorname{Fr}=\frac{U}{\sqrt{g H}},
$$

where $U$ is the mean flow velocity, $g$ is the acceleration due to gravity, and $H$ is the depth of the flow. Of particular interest is the drag acting on obstacles in the flow (or equivalently the force acting on the flow), as this is important for analyzing the performance of structures in a flood, or for calculating the effect of obstacles on the flow itself. The drag force $F_{D}$ is commonly expressed as

$$
F_{D}=C_{D} F_{P} \text { with } F_{P}=\frac{1}{2} \rho U^{2} D H,
$$

where $C_{D}$ is the drag coefficient, $\rho$ is the fluid density, and $D$ is the obstacle width facing the flow direction. Similarly, the standard deviation of the drag force, $F_{D \text { rms }}$, is a useful measure of temporally fluctuating drag forces, which can be quantified in terms of the root-mean-square drag coefficient $C_{D \text { rms }}$ :

$$
F_{D \text { rms }}=C_{D \text { rms }} F_{P}
$$

For developed normal flow in channels the balance between drag and gravity can be expressed in terms of Manning's formula,

$$
U=R_{H}^{2 / 3} S^{1 / 2} n^{-1},
$$

where $R_{H}$ is the hydraulic radius, $S$ is the channel slope, and $n$ is Manning's coefficient [8].

Much previous work has concentrated on flow past circular cylinders. In this case drag coefficients are found to be of order 1, and vary with the Reynolds number Re given by

$$
\operatorname{Re}=\frac{U D}{v},
$$

where $v$ is the kinematic viscosity. For a smooth cylinder, White [9] gives

$$
C_{D}=1+10 \mathrm{Re}^{-2 / 3} \text { for } 1<\operatorname{Re}<O\left(10^{5}\right) .
$$

At higher Re, delayed flow separation reduces $C_{D}$, which is sensitive to Re, surface roughness $k_{s}$, and turbulence intensity $I_{T}$. The drag coefficient is also influenced by wall constraints. If the blockage ratio $D / B$ is large, sidewalls prevent the flow passing the cylinder from expanding laterally, causing it to accelerate and resulting in a compensating fall in pressure. Laboratory measurements on isolated cylinders are often corrected to estimate $C_{D}$ in an infinitely wide channel $C_{D c}$. A variety of methods exist in the literature (e.g., [11-13]) with their efficacy dependent on the Reynolds number regime [10].

Maskell [11] developed a blockage correction procedure, for both bluff and streamlined bodies, based on an approximate momentum balance outside the wake and two empirical auxiliary relations. The final form of the correction is

$$
\frac{C_{D}}{C_{D c}}=1+\varepsilon \frac{D}{B},
$$

where $\varepsilon$ is an experimentally determined coefficient, equal to 0.96 for flat plates. Awbi [12] proposed a modification to Maskell's theory for obstacles with a rectangular cross-section where $\varepsilon$ is a function of the ratio of the cross-sectional dimensions of the obstacle. For square cross sections the reduction is only 3\%, suggesting Maskell's original value is reasonable. Anthoine et al. [10] showed that Maskell's theory is valid for circular cylinders (at subcritical Reynolds numbers, i.e., $\operatorname{Re}<3 \times 10^{5}$ ) even for large blockages (tested for $D / B \leqslant 17 \%$ ). Ranga Raju and Singh [13] proposed an alternative correction procedure for square cross-section obstacles, as well as triangular prisms and circular disks, which was validated for $D / B \leqslant 24 \%$ :

$$
C_{D c}=C_{D}\left(1-\frac{D}{B}\right)^{\alpha}
$$


where $\alpha$ is a function of the ratio of obstacle cross-sectional dimensions, with $\alpha \approx 1.15$ for square obstacles. These correction procedures are therefore reasonable to employ in the present study, given that they have been successfully applied to various bluff body shapes, including square obstacles at high blockage ratios.

For circular cylinder pairs, $C_{D}$ is a function of $s_{x}$ and $s_{y}$, which are the separation between obstacle centers in the streamwise and cross-stream directions respectively. For tandem arrangements, where one cylinder is aligned behind the other in the direction of the mean flow $\left(s_{y}=0\right)$, the drag on the downstream cylinder is substantially reduced due to decreased velocities and increased turbulence in the upstream cylinder wake and thus in flow approaching the downstream cylinder. This effect is known as "sheltering" [14]. Liu et al. [15] found that, for low $I_{T}(0.7 \%)$, flow around smooth cylinders at $\mathrm{Re}=80000, C_{D}$ (for the downstream cylinder) is negative at small separations and suddenly increases at a separation of $s_{x}=3.5 \mathrm{D}$. This behavior disappeared as the surface was roughened or turbulence was introduced into the inflow. For side-by-side arrangements $\left(s_{x}=0\right)$, if the cross-stream separation is sufficiently large $\left(s_{y}>2 D\right)$, the flow pattern is symmetrical relative to the axis passing through the middle of the gap between cylinders [16]. However, at small separations the gap flow is biased to one side, switching between the two sides at irregular intervals. One cylinder is subject to a narrow wake (low drag) and the other to a wide wake (high drag). Zdravkovich and Pridden [16] found that the sum of the high and low drag is always less than twice that on an isolated cylinder, hence interference reduces $C_{D}$ at close spacing. They also found that for a given separation the minimum $C_{D}$ often occurs when the obstacles are offset in both directions $\left(s_{x} \neq 0\right.$ and $\left.s_{y} \neq 0\right)$, known as staggered arrangements, because the gap flow between cylinders shifts the stagnation point of the downstream cylinder (by up to $30^{\circ}$ ), exposing a large area on the front of the cylinder to low pressure.

In arrays of circular cylinders, $C_{D}$ is dependent on $\mathrm{Re}$ and solid volume fraction $\lambda$ (the ratio of the total solid volume to the total volume of solid and fluid). Tanino and Nepf [6] found that in random arrays $C_{D}$ decreases with increasing $\mathrm{Re}$ (where $\mathrm{Re}$ is based on the mean velocity within the array, taking account of the average reduction in cross-sectional area) and increases with increasing $\lambda$ for $25<\operatorname{Re}<685$ and $0.091<\lambda<0.35$. At higher Re the opposite trend $\left(C_{D}\right.$ decreasing with increasing $\lambda$ ) has been reported, for example in the numerical model of Nepf [5] where $C_{D}$ of individual cylinders is assigned based on the position of the closest upstream cylinder $\left(8 \times 10^{-4}<\lambda<0.24\right.$, random and staggered $)$, validated with laboratory results at $\operatorname{Re}>1000$. Cheng and Nguyen [7] introduced the concept of vegetation hydraulic radius, $r_{v}$ (the ratio of the volume of water to the combined frontal area of all cylinders), to collapse laboratory data to a function of a single variable. They show that $C_{D}$ decreases monotonically with $\operatorname{Re}$ (defined in terms of mean array velocity and $r_{v}$ ) and is independent of $\lambda$ (with no significant difference between random and staggered arrays).

Vegetation in environmental flows is not so well represented by the idealized models or laboratory experiments. Values of $I_{T}$ are generally higher, and vegetation is often not circular at all in cross section ("sedges have edges"), and certainly not perfectly circular or smooth. As a result, drag coefficients for real vegetation are typically $C_{D} \sim 2$ and there is less variation with Re. James et al. [17] found in a flume study that isolated stems of Phragmites australis (common reed) have higher drag coefficients than circular cylinders, with square obstacles forming a better model. They also found $C_{D}$ increased further if foliage was present. In a further flume study, Wunder et al. [18] found that for leafy willows at high velocities $C_{D}$ exceeds that of circular cylinders. Thus square obstacles, with fixed separation points and less dependence on Re, can form better models of real vegetation in terms of the magnitude of fluid drag.

Flows past square cross-section obstacles have been less intensively studied, limited mostly to single square obstacles, or special arrangements of pairs of obstacles (tandem or side by side). The value of $C_{D}$ for a single obstacle has been measured by various authors with typical values of the order of 2 (see Table I). Lyn et al. [19] used laser-Doppler velocimetry to measure wake recovery in a closed water channel at $\mathrm{Re}=21400$ and found $C_{D}=2.1$ from the integral of mean velocity profiles (and found the length of the recirculation region was about 1.4D). Yen and Yang [3] calculated $C_{D}$ 
TABLE I. Drag coefficients for square obstacles from previous studies.

\begin{tabular}{lccccc}
\hline \hline Author & $\operatorname{Re}$ & $I_{T}(\%)$ & $D / B(\%)$ & $H / D$ & $C_{D}$ \\
\hline Norberg [20] & 5000 & 0.06 & 1.1 & 62.5 & 2.21 \\
Yen and Yang [3] & 6300 & 0.3 & 4.0 & 25.0 & 1.86 \\
Norberg [20] & 13000 & 0.06 & 1.1 & 62.5 & 2.15 \\
Yen and Liu [4] & 21000 & 0.4 & 4.0 & 25.0 & 2.06 \\
Lyn et al. [19] & 21400 & 2 & 7.1 & 9.8 & 2.1 \\
Yen and Yang [3] & 24000 & 0.3 & 4.0 & 25.0 & 1.96 \\
Yen and Yang [3] & 36000 & 0.3 & 4.0 & 25.0 & 2.02 \\
Lee [21] & 176000 & 0.5 & 3.6 & 9.2 & 2.04 \\
Lee [21] & 176000 & 4.4 & 3.6 & 9.2 & 1.98 \\
Lee [21] & 176000 & 6.5 & 3.6 & 9.2 & 1.94 \\
Lee [21] & 176000 & 8 & 3.6 & 9.2 & 1.81 \\
Lee [21] & 176000 & 12.5 & 3.6 & 9.2 & 1.53 \\
British Standards Institution [22] & N/A & N/A & N/A & N/A & 2.1 \\
\hline \hline
\end{tabular}

from pressure transducer measurements in an open-loop wind tunnel and found $C_{D}$ increased with $\operatorname{Re}$ from 1.86 at $\mathrm{Re}=6300$ to 2.02 at $\mathrm{Re}=36000$. Lee [21] investigated the influence of $I_{T}$ on $C_{D}$ in a low speed wind tunnel. The turbulence level was varied by using four different square mesh grids upstream of the obstacle and $C_{D}$ was obtained from the integrated mean pressure distribution measured using 18 and 6 pressure taps on the front and rear faces respectively. A reduction in $C_{D}$ was observed with an increase in $I_{T}$. This was ascribed to the fact that additional turbulence thickens shear layers which are then deflected by the obstacle's rear corners, causing the vortices to form further downstream. The measured drag coefficients are in the range obtained by other researchers at lower $I_{T}$, for $I_{T}$ up to $6.5 \%$, but then fall below this range as $I_{T}$ increases to $8 \%$, reaching the lowest value of 1.53 at the highest turbulence intensity of $12.5 \%$. In a channel of finite width $B$, increases in blockage ratio $D / B$ would be expected to contribute to an increase in the drag.

For square-section obstacles, an important parameter is the angle of attack $\theta$, i.e. the angle between the mean flow direction and the upstream face of the obstacle [3,20,21]. Yen and Yang [3] investigated the influence of $\theta$ on $C_{D}$ (defined in terms of the projected area which varies with $\theta$ ) and flow patterns visualized using particle image velocimetry (PIV). Three flow modes were identified. In the leading-edge separation mode $\left(\theta<9^{\circ}\right)$ stagnation occurs at the midsection of the front face and the flow divides into two streams. Flow separates near the front vertices and two vortices form in the wake. A local maximum in $C_{D}$ occurs at $0^{\circ}$ due to the high pressure near the stagnation point. As $\theta$ increases, a vortex shed from the front corner intermittently touches the rear corner, reducing $C_{D}$. In the separation bubble mode $\left(9^{\circ}<\theta<27^{\circ}\right)$ flow separates at the leading edges, reattaches on the surface on one side, and separates again at the rear corner with the minimum $C_{D}$ occurring at $12^{\circ}(1.35$ at $\operatorname{Re}=6300$ and 1.50 at $\operatorname{Re}=36000)$. In the attached mode $\left(27^{\circ}<\theta<45^{\circ}\right)$ streamlines near the front vertices smoothly follow the obstacle shape and separation does not occur until the rear vertices. Increases in $\theta$ gradually broaden the wake, increasing $C_{D}$ towards the maximum value at $45^{\circ}$. The present study is restricted to $\theta=0^{\circ}$.

For side-by-side square obstacles Yen and Liu [4] (using pressure transducer and velocity measurements in an open-loop wind tunnel) identified three distinct modes of flow behavior. When the obstacles are sufficiently close $\left(1 \leqslant s_{y} / D \leqslant 1.1\right)$ "single mode" flow behavior is similar to that around an isolated obstacle, but the wake is wider so $C_{D}$ is higher (maximum $C_{D}=2.24$ ). As $s_{y} / D$ increases ("gap-flow mode" $1.1 \leqslant s_{y} / D \leqslant 6.5$ ) jet flow develops between the obstacles, with no flow separation on the interfacial surfaces, so $C_{D}$ is reduced (minimum $C_{D}=1.68$ ). As $s_{y} / D$ increases further ("couple vortex-shedding mode" $6.5 \leqslant s_{y} / D \leqslant 13$ ), interactions are minimal and $C_{D}$ is almost independent of $s_{y} / D\left(C_{D}=2.08\right)$. Yen and Liu [4] also measured $C_{D \text { rms }}$ on an isolated obstacle and the average root-mean-square drag coefficient for each obstacle in a pair. 
The maximum value of 0.263 occurred in the gap flow mode at $s_{y} / D=1.5$, and $C_{D \text { rms }}$ in the couple vortex-shedding mode was 0.241 , close to isolated obstacle value of 0.242 . For square obstacles in tandem, Yen et al. [2] identified (in experiments in a vertical water tank) three distinct modes of flow behavior depending on Re and $s_{x} / D$. When the obstacles are sufficiently close, flow patterns are similar to that of an isolated obstacle ("single mode"). As the separation increases, flow reattaches on the downstream obstacle, resulting in a substantial reduction in downstream obstacle $C_{D}$ ("reattached mode"). At a separation of $3 D, C_{D}$ tends to 0.5 for $\operatorname{Re}>670$. At large separations similar structures form in the wakes of the two obstacles and downstream $C_{D}$ increases towards the isolated value ("binary mode"). The spacing at which the transition between the different flow modes occurs was found to decrease with an increase in Re, and the single mode was only observable for Re up to the order of 600 . A number of researchers have identified a sudden change in $C_{D}$ which corresponds to the transition between the reattached and binary modes (e.g., [23,24]). Kim et al. [24] used PIV to investigate the mean velocity and turbulent statistics of the flow field surrounding tandem pairs of square obstacles for $1.5 \leqslant s_{x} / D \leqslant 11.0$ and $\operatorname{Re}=5300$ and 16000 . A discontinuous jump occurred simultaneously in $C_{D}$ for both obstacles at the critical spacing of $3.5 D$, which was independent of Re within the range studied, and the flow patterns at $s_{x} / D \leqslant 3.0$ were drastically different from those at $s_{x} / D \geqslant 3.5$.

A number of researchers have also employed numerical models to determine drag on square obstacles (e.g., [25-27]). Rodi [25] conducted a comprehensive study comparing various Reynoldsaveraged Navier-Stokes (RANS) and large eddy simulation (LES) models for the test case with a single obstacle presented by Lyn et al. [19] $\left(C_{D}=2.1\right)$. For the RANS simulations, $C_{D}$ was well predicted by the $k$-epsilon model with the Kato-Launder modification and a two-layer approach to resolving the near-wall region (2.0) and by a Reynolds-stress model with wall functions (2.15). LES models generally gave a better simulation of the details of the flow at the expense of a large increase in computation time, but there were large differences between individual calculations, particularly in terms of $C_{D}$ which varied between 2.02 and 2.77, which the author noted were difficult to explain. The literature concerning simulations of the flow past obstacle pairs is far more sparse, but numerical models have been employed to investigate the flow around tandem pairs albeit at low Reynolds numbers (using 2D incompressible laminar flow models) (e.g., [26,27]). Sohankar and Etminan [26] considered obstacles separated by $s_{x} / D=6$ at $1 \leqslant \operatorname{Re} \leqslant 200$ and $D / B=5 \%$, using both steady and unsteady models. The results demonstrate that the flow becomes unsteady for $\operatorname{Re} \geqslant 40$, and for $\mathrm{Re}=50$ pressure drag contributes to $80 \%$ of the total drag. For Re $\geqslant 50$ upstream $C_{D}$ is relatively constant, whereas the downstream obstacle $C_{D}$ increases gradually due to the change of flow pattern between the obstacles. For Re $\geqslant 100$, the downstream obstacle $C_{D}$ also becomes approximately constant, with $C_{D}$ equal to 1.53 and 1.30 for the upstream and downstream obstacles respectively at $\mathrm{Re}=100$. Lankadasu and Vengadesan [27] studied the flow around tandem obstacles subject to both uniform and planar shear inflow, for $2 \leqslant s_{x} / D \leqslant 7$ and $\mathrm{Re}=100$. The results showed that, with an increasing shear gradient, the flow became unsteady at lower $s_{x} / D$. When $s_{x} / D>4$, there was a clearly defined wake behind the upstream obstacle, but below this threshold there was no definite wake, despite the fact that the flow was unsteady for some combinations of $s_{x} / D$ and the shear gradient. The downstream obstacle $C_{D}$ was consistently lower than that of the upstream obstacle and was far lower than that of the single obstacle in uniform flow. Negative values were obtained at lower $s_{x} / D$ for uniform flow or low shear gradients, but $C_{D}$ became positive as the shear gradient increased. The root-mean-square drag coefficient was consistently higher for the downstream obstacle than the upstream one. With increasing $s_{x} / D, C_{D \text { rms }}$ initially increased and then began to decrease for the upstream obstacle, whereas it increased monotonically for the downstream one. Accurate estimation of $C_{D}$ for pairs of obstacles at high Reynolds numbers remains challenging.

Here we will use laboratory flume experiments to measure the drag on a pair of square obstacles in a comprehensive set of arrangements covering the range $-5 \leqslant s_{x} / D \leqslant 20$ and $0 \leqslant s_{y} / D \leqslant$ 3.5 (or 7.0 for $D / B=6.3 \%$ ). Previous work only considered special cases with perpendicular alignment between the obstacles (tandem and side-by-side arrangements), whereas we extend this to the general case including other, nonperpendicular relative positions. In Sec. II we describe the 


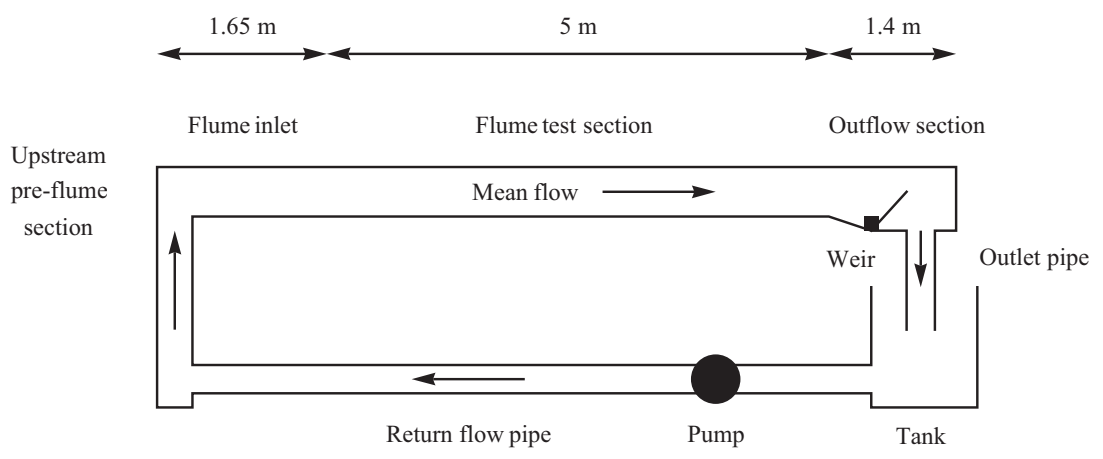

FIG. 1. Sketch of the flume used for the experiments.

experiments and give measurements of the flume flow without obstacles. Next, in Sec. III, we present the results including the main features of the velocity field with obstacles present and comprehensive results for the drag on pairs of obstacles. Finally, in Sec. IV, we discuss the results and present our main conclusions.

\section{EXPERIMENTS}

The experiments were conducted in a recirculating tilting flume, with main working section of length $5 \mathrm{~m}$, and width and height $300 \mathrm{~mm}$ (Fig. 1). The cross section of the upstream pre-flume section contracts just upstream of the flume inlet, but there are no other means of turbulence suppression. Downstream of the flume test section there is an outlet with an adjustable overflow weir, which is controlled by a wheel. The maximum flow rate is approximately $Q=0.03 \mathrm{~m}^{3} \mathrm{~s}^{-1}$.

The flow rates were adjusted after obstacles were placed in the water to give subcritical flow, with Fr $<0.59$ in all cases, and the slope and weir height were adjusted to obtain uniform flow in the test section. To calibrate the slope, the flume was filled with water and both ends of the test section were sealed. From repeated measurements of the depth of still water, an accuracy of $\pm 0.3 \mathrm{~mm}$ was achievable. The uncertainty in the slope $S$ is therefore $\pm(2 \times 0.3 / 5000)=$ $\pm 1.2 \times 10^{-4}$. Velocities were measured using a Nortek Vectrino ADV operating at $200 \mathrm{~Hz}$. The accuracy of velocity measurements is $\pm 0.5 \%$ of the measured value $\pm 0.001 \mathrm{~ms}^{-1}$ and the Doppler uncertainty is estimated as $1 \%$ of the user-specified nominal velocity range [28]. This range was adjusted to minimize uncertainty while capturing the entire range of velocities. It was typically set to $\pm 1 \mathrm{~ms}^{-1}$ but up to $\pm 4 \mathrm{~ms}^{-1}$ was necessary in the wake of an obstacle. The maximum uncertainty is therefore $\pm(0.041+0.005 U) \mathrm{ms}^{-1}$. The ADV probe was fully submerged when measuring velocities surrounding obstacles. For the measurement of velocities in the channel with no obstacles present, the ADV probe was fully submerged except at the maximum height where only the transmitter and bottom two (of four) receivers were submerged. This is acceptable for the measurement of the streamwise component (as well as the lateral component but not the vertical one). See Robertson [29] for further experimental details.

Mean streamwise velocity profiles, nondimensionalized by the bulk mean speed $U=Q /(B H)$, in the absence of obstacles are shown in Fig. 2. The velocity profiles are consistent with the logarithmic law near the base of the flume. Further from the base, in the free stream, the velocity remains constant with respect to depth. This is true above $\sim H / 2$ or $\sim 8 \mathrm{~cm}$ from the base, so velocities either side of obstacles were measured above this height. The increase in free-stream velocity with downstream distance is relatively small, especially when compared to that downstream of an obstacle (see below). The velocity profile in the cross-stream direction is reasonably uniform. The velocity decreases slightly towards the center of the flume and towards the walls. The turbulence intensity varies with position but is of the order of $10 \%$. Manning's coefficient was found to be $n=$ 

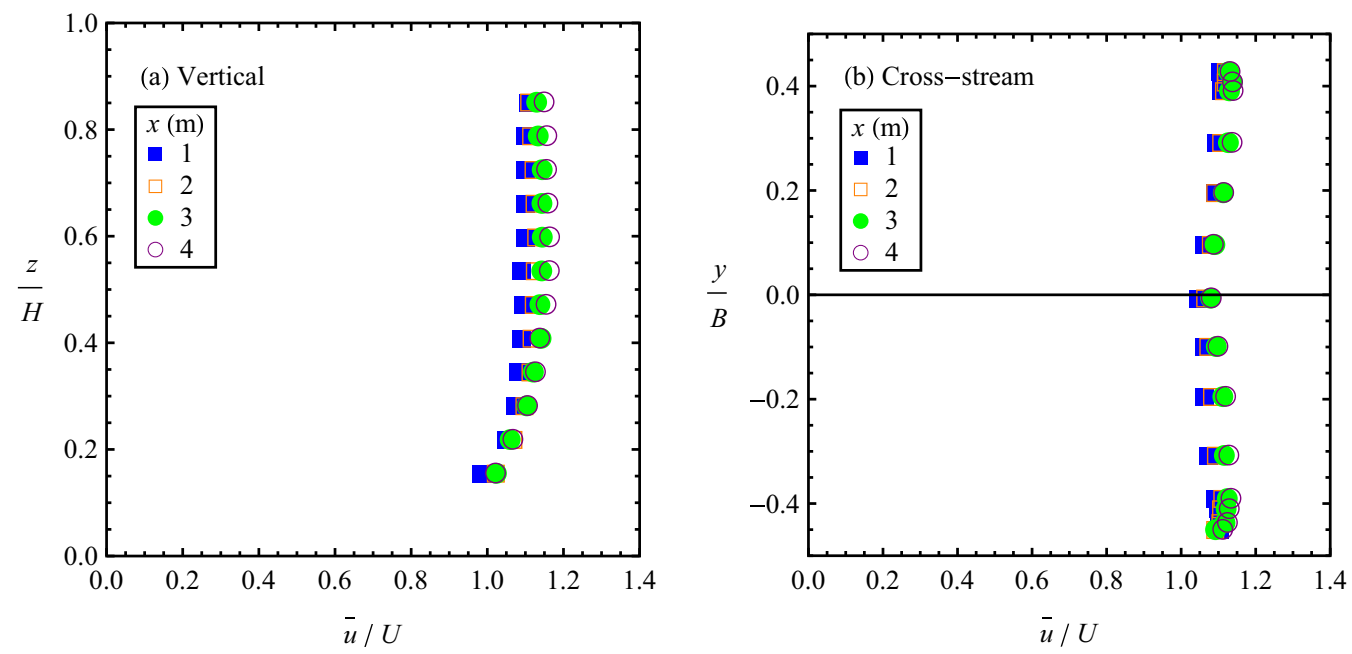

FIG. 2. Profiles of the normalized, temporally averaged, streamwise component of velocity in the flume with no obstacles present, at different downstream distances from the start of the main working section $x$ for $Q=0.0166 \mathrm{~m}^{3} \mathrm{~s}^{-1}, H=158 \mathrm{~mm}$, and $S=0.0007$. (a) In the vertical direction at the center of the channel and (b) in the cross-stream direction at a height of $H / 3$ below the free surface.

$0.011 \mathrm{~m}^{-1 / 3} \mathrm{~s}$. This was computed from linear regression of $U$ against $R_{H}{ }^{2 / 3} S^{1 / 2}$ at $S=0.0013$ and 0.0022 over a range of conditions where $0.0142 \leqslant Q \leqslant 0.0275 \mathrm{~m}^{3} \mathrm{~s}^{-1}$ and $0.093 \leqslant H \leqslant 0.196 \mathrm{~m}$.

Two sizes of obstacle were used with $D=38 \mathrm{~mm}$ and $19 \mathrm{~mm}(D / B=12.7 \%$ and $6.3 \%)$ always placed square on to the flow. The obstacles are made from aluminum which has a surface roughness of $k s=1-2 \mu \mathrm{m}$ [30]. Consulting a Moody chart, the obstacles can therefore be considered hydraulically smooth since the upper estimate of $k s / D$ is of the order of $10^{-4}$ and the maximum $\operatorname{Re}$ (based on obstacle width) is of the order of $2 \times 10^{4}$.

Forces on the obstacles were measured by a strain gauge recording data at $200 \mathrm{~Hz}$, calibrated before and after each set of experiments by applying known loads. The difference between an applied load and that computed from calibration constants is negligible. The best fit consistently gave a correlation coefficient $R^{2}>0.999$. The uncertainty of the strain gauge measurements was estimated as $\pm\left(0.02+0.01 F_{D}\right) \mathrm{N}$. This accounts for a potential shift in calibration constants over the course of an experiment as well as the difference between the mean drag determined over the measurement period of $120 \mathrm{~s}$ and over a much longer period. When the calibration was checked at the start of each experiment the difference between known and measured forces was less than $2 \%$ or the gauge was recalibrated. However, this may underestimate the uncertainty due to calibration drift at low forces as, for the check, applied loads were greater than or equal to $0.981 \mathrm{~N}$, but in the experiments $F_{D} \geqslant 0.2 \mathrm{~N}$, so the uncertainty was estimated as $2 \%$ of $0.981 \mathrm{~N}$. Measurements were discarded from further analysis if there was a difference of over $2 \%$ between forces computed with the calibration constants obtained between successive calibrations. In preliminary tests, the mean value of $C_{D}$ was calculated over increasing periods of time, $t$. This mean value remained constant to within $1 \%$ for $t>120 \mathrm{~s}$, so this period was used for calculating the mean value of $C_{D}$.

A sketch showing the arrangement with two obstacles is shown in Fig. 3. One obstacle was kept centrally positioned, mounted on the strain gauge, while a second was placed in different positions to vary the relative positions, with $s_{x}$ and $s_{y}$ being the position of the gauged obstacle relative to the second obstacle. Negative values of $s_{x}$ indicate that drag was measured on the upstream obstacle. Keeping the obstacle attached to the strain gauge stationary minimized the uncertainty and recalibration that would have been required if this obstacle had been moved. The upstream distance from the obstacles to the start of the working section was always greater than $61 D$ while 


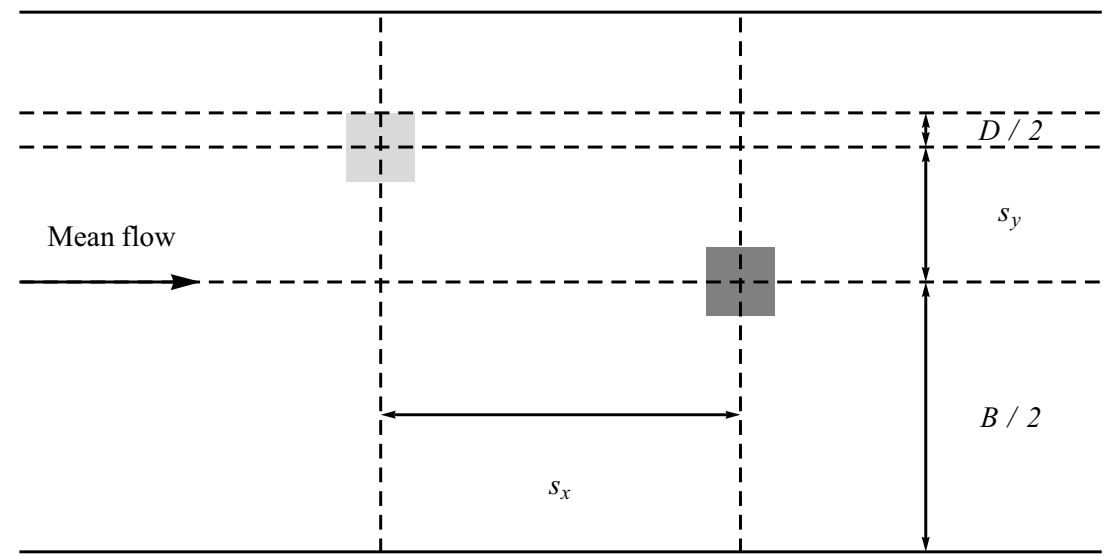

FIG. 3. Sketch showing the arrangement of obstacles (here with $D / B=12.7 \%$ ). The darker obstacle is mounted on a strain gauge in the center of the channel, while the lighter obstacle is moved to different positions. A negative value of $s_{x}$ indicates that the drag is measured on the upstream obstacle.

the distance downstream of the obstacles to the end of the working section was always greater than $46 D$. There is a small gap $(<2 \mathrm{~mm})$ between the obstacle attached to the strain gauge and the base of the flume. Other obstacles are in contact with the channel floor. For single obstacle experiments, only the gauged obstacle is present.

Some preliminary experiments were conducted with a single obstacle. This was followed by experiments with special arrangements of two obstacles ("tandem pair," $s_{y}=0$, and "side-byside pair," $s_{x}=0$ ) and finally experiments with a range of relative positions ("staggered"). The conditions for these experiments are summarized in Table II.

A nominal flow rate of $0.0167 \mathrm{~m}^{3} \mathrm{~s}^{-1}$ was used for each of the experiments conducted under fixed flow conditions (isolated obstacle velocity, tandem pair velocity, and staggered pair drag measurements), with uniform depth throughout the test section, and $C_{D}$ was computed as the ratio of $F_{D}$ and $F_{P}$. When measuring the drag over a range of conditions (isolated obstacle and tandem pair drag measurements) $Q, H$, and $S$ were all varied. The depth was not always uniform throughout the entire test section but there was no change in depth across the obstacle(s). For isolated obstacles $0.0064 \leqslant Q \leqslant 0.0302 \mathrm{~m}^{3} \mathrm{~s}^{-1}$ and for tandem obstacles $0.0087 \leqslant Q \leqslant 0.0239 \mathrm{~m}^{3} \mathrm{~s}^{-1}$. The mean drag coefficient was estimated from linear regression of $F_{D}$ vs $F_{P}$. A minimum of five data points were analyzed and $R^{2}>0.98$ for all cases.

TABLE II. Summary of main experimental conditions for the different types of experiments.

\begin{tabular}{lccccccc}
\hline \hline Obstacle(s) & Measurements & Fig(s). & $I_{T}(\%)$ & $S$ & $H / D$ & Fr & $\operatorname{Re}$ \\
\hline Isolated obstacle & Velocity & 4 and 9 & $\approx 10$ & 0.0022 & 4.9 & 0.22 & 11000 \\
Isolated obstacle & Drag & 5 and 6 & & $0.0007-0.0022$ & $1.4-6.0$ & $0.21-0.59$ & $9500-21900$ \\
Tandem pair & Velocity & 7 & $\approx 8$ & 0.0007 & 3.3 & 0.38 & 16000 \\
Tandem pair & Drag & 8 & & $0.0007-0.0022$ & $2.7-6.3$ & $0.08-0.33$ & $4800-16500$ \\
Staggered pair & Drag & $8-12$ & $\approx 8$ & 0.0007 & 3.3 & 0.38 & 16000 \\
Staggered pair & Drag & $8,10-12$ & $\approx 8$ & 0.0007 & 7.7 & 0.33 & 7500 \\
\hline \hline
\end{tabular}

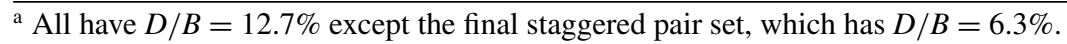




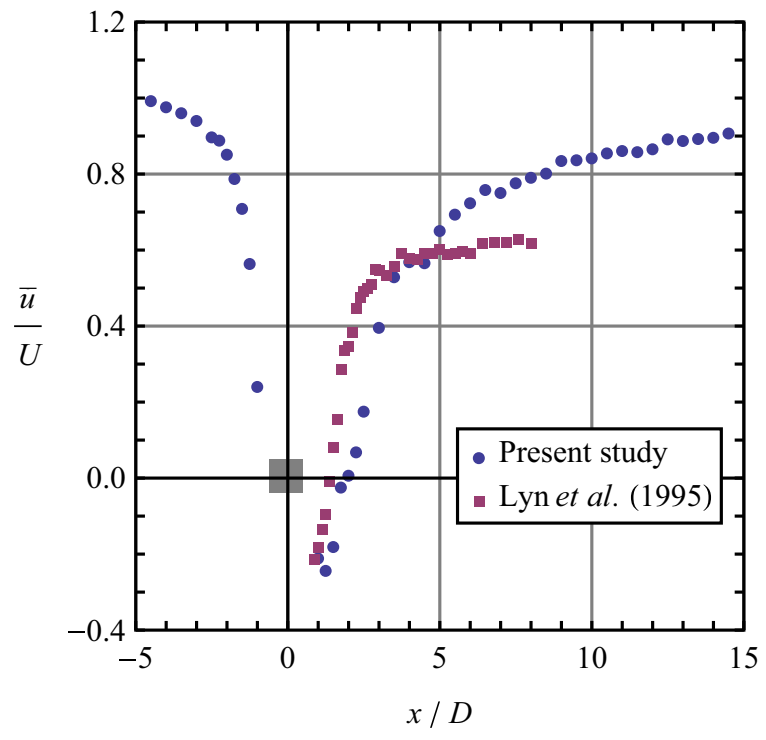

FIG. 4. Normalized, temporally averaged, streamwise component of velocity on the channel centerline at a depth of $H / 3$ below the free surface for flow past a single obstacle with $D / B=12.7 \%$ and $\operatorname{Re}=11000$. The results from Lyn et al. [19] are also shown.

\section{RESULTS AND ANALYSIS}

\section{A. Isolated obstacles}

The mean streamwise velocities on the channel centerline at a depth of $H / 3$ below the free surface, nondimensionalized by the velocity $10 \mathrm{D}$ upstream (1.0\% higher than the bulk velocity), are shown in Fig. 4 for the preliminary experiments with a single obstacle. The turbulence intensity $10 D$ upstream is $10 \%$. Velocities are shown both upstream and downstream of the obstacle, and compared with the results from Lyn et al. [19]. In the present experiments, the velocity decreases rapidly upstream of the obstacle and is negative until $2.0 \mathrm{D}$ downstream. The flow recovers to $90 \%$ of its initial value by approximately $14 D$ downstream of the obstacle. In the results of Lyn et al., the wake recovery is initially more rapid with the transition to positive velocity at $1.4 D$. However, the wake does not appear to fully recover in the Lyn et al. experiments, at least within the zone measured (to $8 D$ ), and appears to plateau at $\sim 60 \%$ of the free-stream value. It is possible that the wake is affected by the downstream boundary conditions, while wake recovery is also likely to be affected by the blockage ratio and turbulence intensity (in the present study $D / B=12.7 \%$ and $I_{T}=10 \%$, whereas for Lyn et al. $D / B=7.1 \%$ and $I_{T}=2 \%$ ). In the present study, the wake diffuses as it moves downstream and meets the tank boundary layer. The more complete wake recovery in the present experiments gives confidence that our results are not strongly influenced by downstream boundary conditions.

The drag force on the single obstacle with $D / B=12.7 \%$ was measured for a range of flow conditions, varying the flow depth and Reynolds number. The measured force $F_{D}$ is plotted against $F_{P}$ in Fig. 5, and the measurement uncertainty is also shown. The best fit line gives $C_{D}=2.11 \pm 0.07$ at the $95 \%$ confidence level assuming the uncertainty of the measurements is negligible. This assumption seems reasonable as the maximum $F_{D} \sim 2 \mathrm{~N}$ and the uncertainty in $F_{D}$ is $0.02 \pm 0.01 F_{D}$, giving a maximum value of $0.04 \mathrm{~N}(1.7 \%)$. The typical measurement uncertainty is therefore much lower than the $\pm 0.07(3.3 \%)$ estimate of the maximum difference between $C_{D}=2.11$ and the true mean. This result, together with the results for two comparable cases with $D / B=12.7 \%$ and $6.3 \%$, are given in Table III. These two cases represent the flow conditions used 


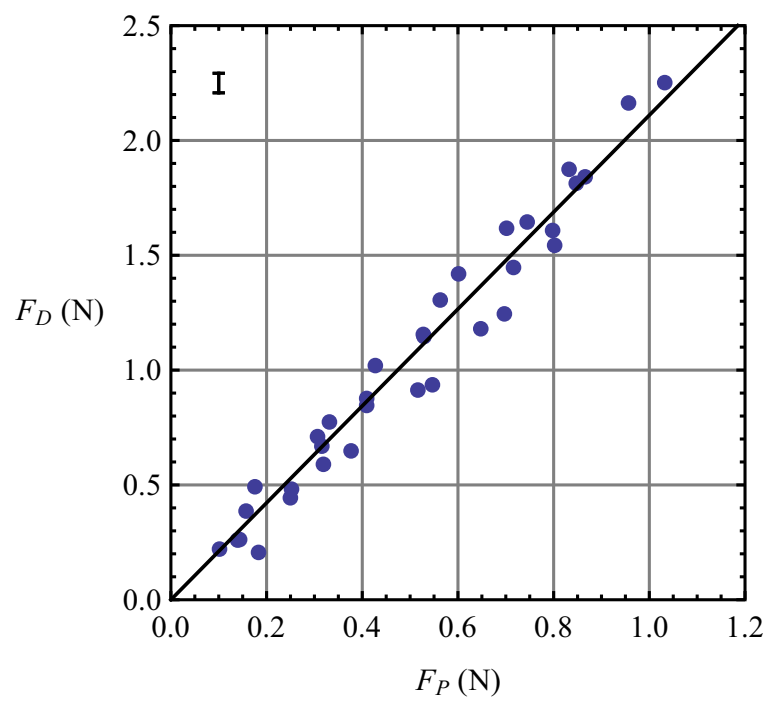

FIG. 5. Measured drag force on a single obstacle plotted against the expected scale for the force. The diagonal line marks the linear regression $\left(C_{D} \approx 2.1\right)$. The vertical line in the top left corner represents the maximum measurement uncertainty in $F_{D}$.

for staggered pair drag measurements and $C_{D}$ was determined using the same method, i.e., the ratio of $F_{D}$ and $F_{P}$ from a single measurement. The turbulence intensity in Table III is taken as the value which was measured at the same $Q$ and $H$, with two obstacles present (see below), at $1 / 3$ depth from the surface and $10 \mathrm{D}$ upstream of the first obstacle. This is assumed to be representative of the upstream $I_{T}$ throughout the free stream.

The results from the present study for the drag coefficient on a single square obstacle are similar to those found by earlier authors (compare Tables III and I). One may anticipate that as the obstacles in this experiment are emergent the pressure drag is accompanied by a resistance due to the formation of surface waves, and that $C_{D}$ would therefore be higher in this experiment than the values measured by other researchers. However, this is not generally true. The comparatively high $D / B$ in this study would also be expected to contribute to a higher $C_{D}$. These effects may be offset by the higher $I_{T}$ in this experiment. There are several other factors which may potentially account for variations in $C_{D}$ including $\mathrm{Re}$ and $H / D$ as well as differences in the experimental setups and measurement techniques. However, from Tables I and III, the value of drag coefficient $C_{D} \approx 2.1$ appears to be relatively insensitive to these differences, when compared to the behavior of $C_{D}$ for circular cylinders. For circular cylinders, the design values of $C_{D}$ given for structures in EUROCODE 1 [22], drop from 1.2 to 0.4 between $\operatorname{Re}=1.81 \times 10^{5}$ and $3.98 \times 10^{5}$, and a similar trend is given by Simiu and Scanlan [31]. Although this occurs slightly outside the range of Re in Tables I and III (up to $1.76 \times 10^{5}$ ), this is due to separation points moving further downstream, which therefore

TABLE III. Results for drag coefficient for a single square obstacle from the present study, with blockage corrections applied according to the methods of Maskell [11] and Ranga Raju and Singh [13].

\begin{tabular}{lcccccc}
\hline \hline $\operatorname{Re}$ & $I_{T}(\%)$ & $D / B(\%)$ & $H / D$ & $C_{D}$ & $C_{D c}[11]$ & $C_{D c}[13]$ \\
\hline $9500-21900$ & & 12.7 & $1.4-6.0$ & 2.11 & 1.62 & 1.66 \\
16000 & $\approx 8$ & 12.7 & 3.3 & 2.06 & 1.65 & 1.76 \\
7500 & $\approx 8$ & 6.3 & 7.7 & 1.79 & 1.68 & 1.80 \\
\hline \hline
\end{tabular}




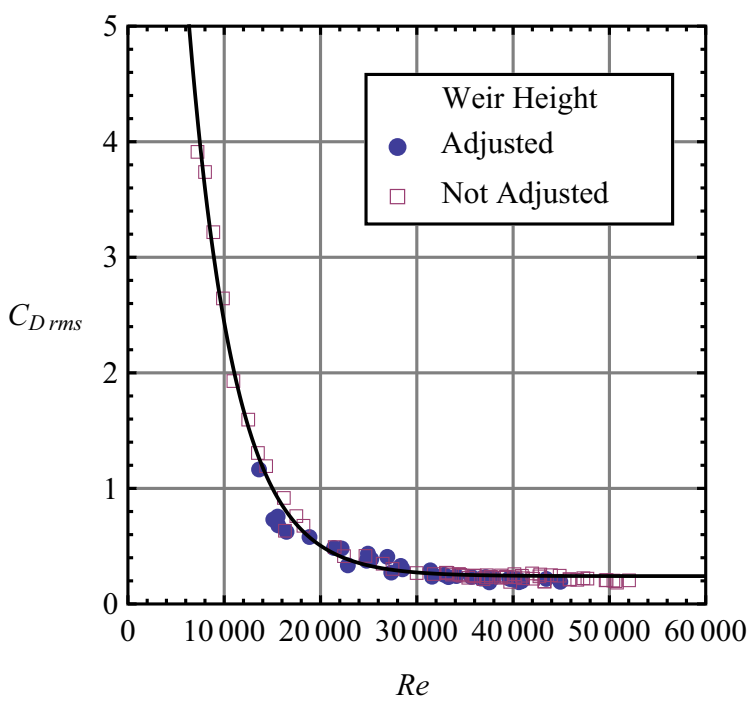

FIG. 6. Root-mean-square drag coefficient of an isolated obstacle at the channel center plotted against Reynolds number, based on the upstream hydraulic radius. The best fit (solid line) is given by Eq. (9).

does not occur for obstacles with a square cross section. The drag coefficient of square obstacles is of the order of 2.0 for Re values higher than in Table I, e.g., between $O\left(2 \times 10^{4}\right)$ and $O\left(2 \times 10^{6}\right)$ according to Simiu and Scanlan [31]. Considering the range of values from Tables I and III, $C_{D}$ drops by $31 \%$, which is significant but much smaller than the $67 \%$ drop observed for circular cylinders over a narrow Re range. Furthermore, this large drop in $C_{D}$ for square obstacles only occurs when $I_{T}$ increases by two orders of magnitude.

Blockage corrections were applied to measurements from the present study and are included in Table III. The corrected values are in keeping with Lee's [21] values for high $I_{T}$ (Table I). Maskell's correction [11] performs better than Ranga Raju and Singh's [13] in collapsing drag coefficients from the present study to a single value.

Figure 6 shows $C_{D \text { rms }}$ for an isolated obstacle as a function of upstream Re, defined in terms of hydraulic radius. To extend the range of Re, additional measurements were included where the height of the weir, downstream of the test section, was not adjusted to obtain a constant flow depth across the obstacle (squares). These measurements are characterized by the upstream conditions $0.05 \leqslant \operatorname{Fr} \leqslant 0.62,1.5 \leqslant H / D \leqslant 6.3$, and $0.0056 \leqslant Q \leqslant 0.0311 \mathrm{~m}^{3} \mathrm{~s}^{-1}$. The upstream Reynolds number, based on $D$, is between 2900 and 26600. The root-mean-square drag coefficient decreases monotonically with $\mathrm{Re}$, and data collapse reasonably well to a single curve suggesting that $C_{D \text { rms }}$ is independent of whether the flow depth is uniform across the obstacle. An appropriate best fit function across both data series is

$$
C_{D \text { rms }}=18.4 e^{-2.13 \times 10^{-4} \mathrm{Re}}+0.241 .
$$

The root-mean-square drag coefficient asymptotically approaches 0.241 at high Re, in close agreement with the value of 0.242 obtained by Yen and Liu [4] at higher $\operatorname{Re}\left(1.5 \times 10^{5}\right)$, suggesting that this is a good approximation beyond the range considered here.

\section{B. Tandem obstacle pairs}

The mean centerline streamwise velocity, nondimensionalized by the velocity $10 D$ upstream (1.2\% higher than the bulk velocity), is shown for two tandem obstacle pairs in Fig. 7, while drag 

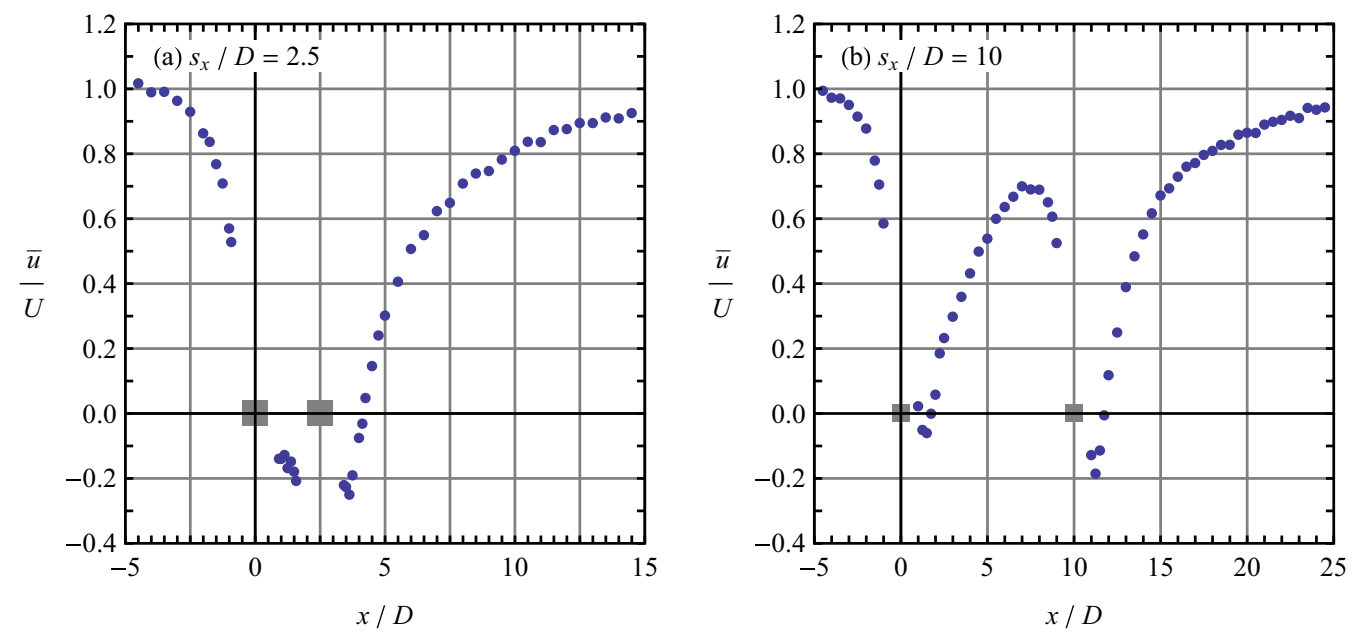

FIG. 7. Normalized, temporally averaged, streamwise component of velocity on the channel centerline for a tandem pair of obstacles with $D / B=12.7 \%, \mathrm{Re}=16000$, and separations between obstacle centers of (a) $2.5 \mathrm{D}$ and (b) $10 \mathrm{D}$.

coefficients for tandem pairs with $-5 \leqslant s_{x} / D \leqslant 20$ are shown in Fig. 8. The turbulence intensity $10 \mathrm{D}$ upstream of the first obstacle is $8 \%$. The velocity upstream of the first obstacle and downstream of the second is close to the isolated obstacle case, independent of $s_{x}$. At close spacing the measured velocities between the obstacles are negative. The flow shown in Fig. 7(a) $\left(s_{x}=2.5 D\right)$ is equivalent to the "reattached mode" identified by Yen et al. [2], where flow reattaches on the downstream obstacle and the downstream drag is substantially reduced. As $s_{x}$ increases the flow between the obstacles recovers, reaching above $70 \%$ of the upstream value when $s_{x}=10 D$ [Fig. 7(b)]. This

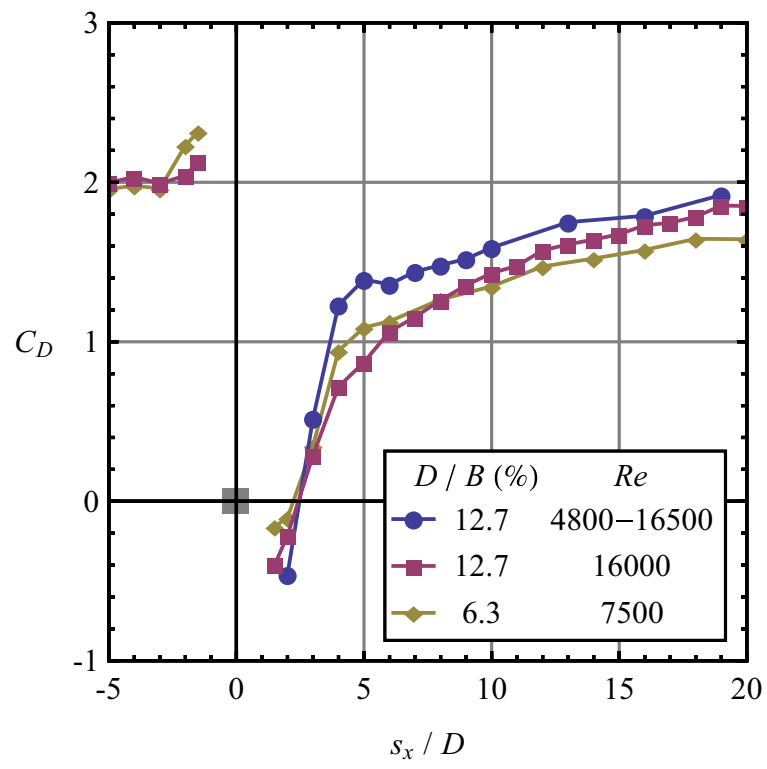

FIG. 8. Drag coefficient for the second obstacle in a tandem pair, positioned $s_{x}$ downstream of the first obstacle (so $s_{x}<0$ indicates the drag is measured on the upstream obstacle). 

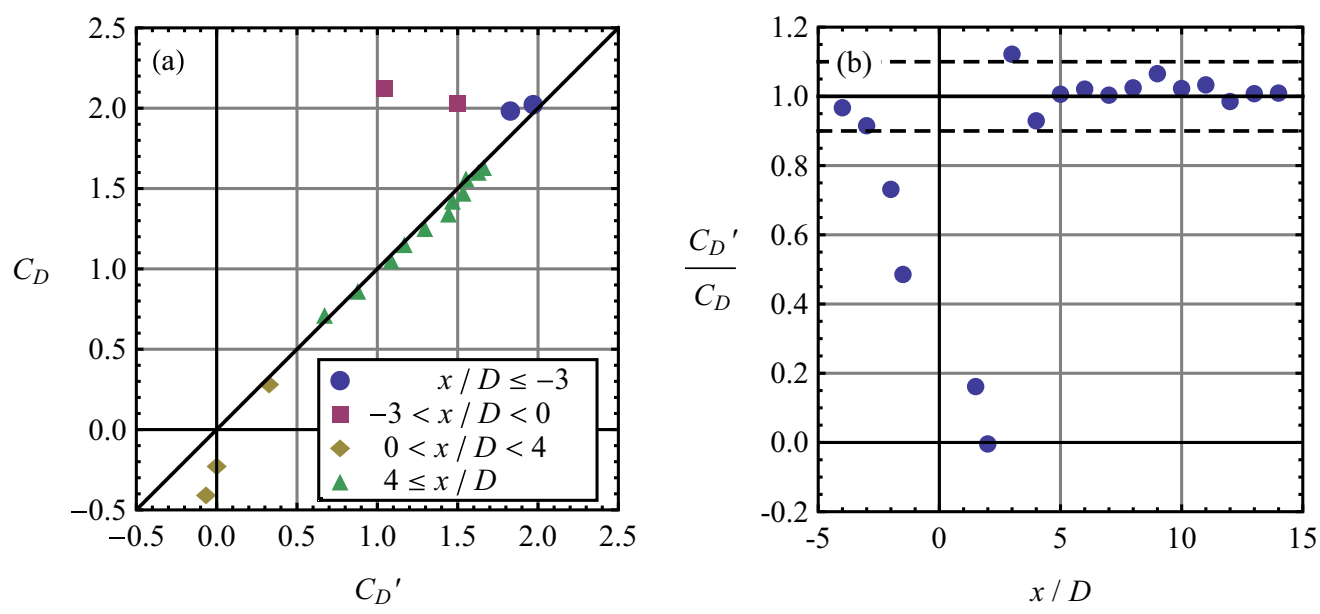

FIG. 9. Comparison of predicted and measured drag coefficients at $D / B=12.7 \%$. The drag was measured at $\operatorname{Re}=16000$ and the local velocity, used to compute $C_{D}{ }^{\prime}$, was measured at $\operatorname{Re}=11000$. The continuous lines represent perfect agreement. (a) The measured drag coefficient plotted against the predicted drag coefficient. (b) The ratio of the predicted and measured drag coefficient plotted against the normalized streamwise distance. Dotted lines enclose an area of agreement between the predicted and measured values to within $\pm 10 \%$.

represents the "binary mode" flow where the velocity recovers to some extent behind both obstacles and the downstream drag coefficient increases towards the isolated obstacle value. Yen et al. found that at $s_{x}=3 D, C_{D}$ tends to 0.5 for $\mathrm{Re}>670$. This is comparable to the values found in the present study (at much higher Re) although $C_{D}$ is very sensitive to position for $s_{x}<4 D$.

Figure 9 assesses to what extent the drag on an obstacle in a tandem arrangement can be predicted by assuming it varies only because of the change in flow speed induced by the other obstacle, taking the induced flow field as being that from a single isolated obstacle. The predicted drag coefficient $C_{D}{ }^{\prime}$ is then the isolated obstacle $C_{D}$ scaled by the local square of the normalized mean streamwise velocity component. The prediction becomes unreliable where $\bar{u}$ (and $C_{D}$ ) changes abruptly across a length comparable to $D$, tending to underestimate the magnitude of $C_{D}$. This suggests that at a distance far enough away from the obstacle the change in streamwise speed is the primary reason for a change in drag, but when the obstacles are closer together the nature of the flow changes, giving a very different drag. The prediction is demonstrated to be reasonably accurate provided that the obstacles are not too close together, as $C_{D}{ }^{\prime}$ is within $\pm 10 \%$ of $C_{D}$ for $x \leqslant-3 D$ and $x \geqslant 4 D$.

\section{Side-by-side obstacle pairs}

Drag coefficients on one obstacle of a pair placed in side-by-side configuration are shown in Fig. 10. Here the drag on the obstacle in the center of the channel is shown, with the other obstacle offset nearer one of the sidewalls. The drag coefficient is consistently higher than an isolated obstacle at the same Re and $D / B$, in contrast to the work of Yen and Liu [4] who found that $C_{D}$ is consistently less than the isolated obstacle value at $\operatorname{Re}=21000$ and $D / B=4 \%$ (except in the single mode $1 \leqslant s_{y} / D \leqslant 1.1$, which is smaller than considered here). Yen and Liu [4] showed $C_{D}$ was independent of $\operatorname{Re}$ for $\operatorname{Re}>17000$. The value of $\operatorname{Re}$ in the present study (for $D / B=12.7 \%$ ) is only $5.9 \%$ lower than this value, suggesting that the difference in behavior is mainly due to the blockage ratio, as well as differences in the experiment setup, as opposed to differences in Re. The blockage ratio is of higher importance in side-by-side configurations, as twice the effective fraction of the cross-section is blocked. In a narrower channel, relative to $D$, the flow around the obstacles is accelerated compared to the flow around the obstacles in a wide channel. Thus, obstacles in a channel with a higher blockage ratio experience a higher drag force. This also explains why 


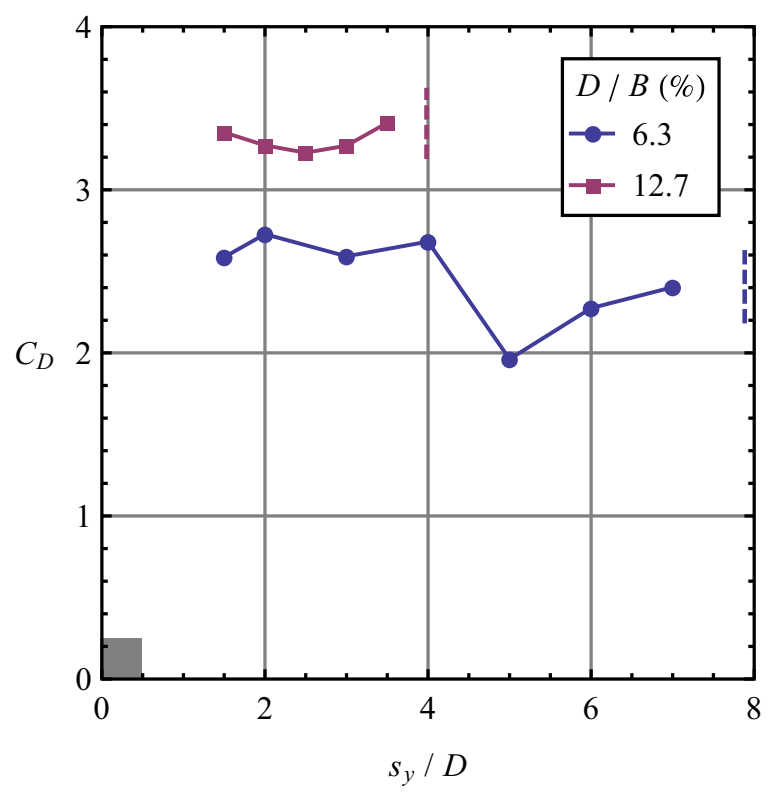

FIG. 10. Drag coefficient on one obstacle of a side-by-side pair. The drag is measured on an obstacle in the center of the channel, while the second obstacle would be against the channel sidewall at $s_{y}=3.5 D$ (for $D / B=12.7 \%$ ) or $7.4 D$ (for $D / B=6.3 \%$ ). The position of the sidewall in each case is indicated by the dashed lines. Reynolds number is 16000 for $D / B=12.7 \%$ and 7500 for $D / B=6.3 \%$.

$C_{D}$ increases with an increase in $D / B$. At $6.3 \%$ blockage, after the initially constant region, $C_{D}$ decreases abruptly towards the isolated obstacle value, but, as $s_{y}$ increases further, one obstacle is placed closer to the flume wall so the flow is diverted towards the test obstacle and $C_{D}$ increases.

\section{Staggered obstacle pairs}

The drag coefficient on a second obstacle at a range of relative positions $\left(-5 \leqslant s_{x} / D \leqslant 20\right.$ and $0 \leqslant s_{y} / D \leqslant 3.5$ or 7.0 ) is shown in Fig. 11, while the same information is presented in the form of contour plots of $C_{D}$ in Fig. 12. When $s_{y}$ is small, the upstream obstacle shields the downstream one from high velocity flow, tending to reduce $C_{D}$. As the obstacles are moved further apart in the $x$-direction shielding is reduced and $C_{D}$ increases towards the isolated obstacle value, reaching $90 \%$ of the isolated value for $s_{x}=19 D$ and $17 D$ respectively at $D / B=12.7 \%$ and $6.7 \%$.

If $s_{y}$ is large and $s_{x}$ is small, twice the effective cross section is blocked so $C_{D}$ is higher than the isolated value. The maximum $C_{D}$ at $D / B=12.7 \%$ blockage is 3.82 , compared to only 2.85 at $D / B=6.3 \%$, and occurs with the obstacles nearly side by side, with the highest drag on the slightly upstream obstacle at $s_{x} / D=-1$. This is presumably because the flow acceleration in the gap between the obstacles in this configuration gives a particularly low pressure at the rear corner of the upstream obstacle, and thus a low pressure in the wake region and on the downstream face of the upstream obstacle, enhancing the total drag force. As $s_{x}$ increases $C_{D}$ decreases towards the isolated obstacle value, but this process is relatively slow.

A plot similar to Fig. 12 for circular cylinders at $\mathrm{Re}=2600$ was presented by Nepf [5]. Measurements were taken for $0<s_{y} / D<2$ and $0<s_{x} / D<5$, and trends further downstream $\left(6<s_{x} / D<20\right)$ were estimated from the observed decay of wake interference. As one may have anticipated, $C_{D}$ is typically much higher for square obstacles. For circular cylinders $C_{D}=1.17$ as $s_{x} / D$ and $s_{y} / D$ approach infinity, similar to the plateau in $C_{D}$ of tandem square obstacles at large $s_{x} / D$. However, for circular cylinders $C_{D}$ never substantially exceeds the isolated cylinder 
(a)

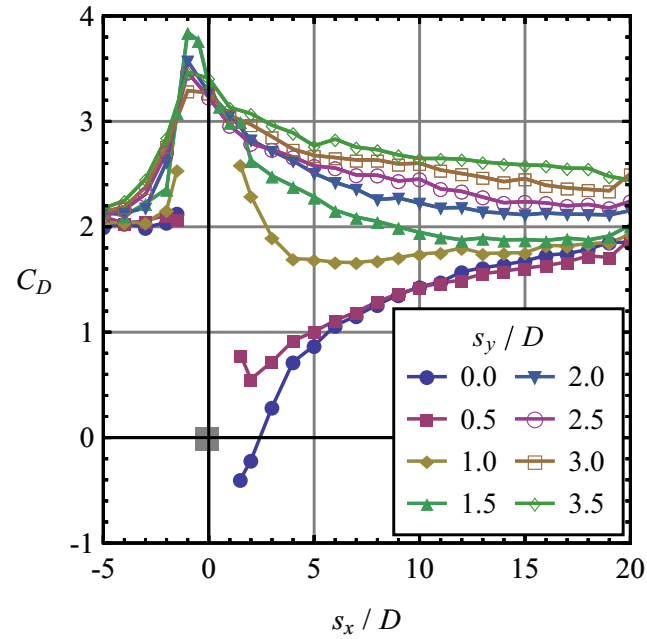

(b)

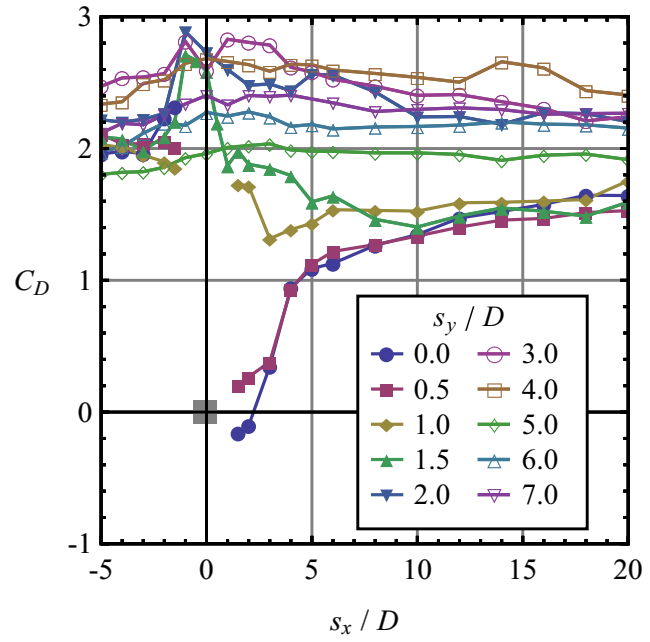

FIG. 11. Drag coefficient on a second obstacle as a function of relative position for (a) $D / B=$ $12.7 \%(\operatorname{Re}=16000)$ and $(b) D / B=6.3 \%(\operatorname{Re}=7500)$.

value, unlike square obstacles where interference and blockage contribute to substantially higher drag coefficients than the isolated obstacle value when $s_{y} / D$ is sufficiently large. The wall position also prevents $C_{D}$ from tending to this value as $s_{y}$ increases. For circular cylinders at $s_{x} / D>2$ the downstream cylinder drag increases as both $s_{x} / D$ and $s_{y} / D$ increase. Similar behavior is observed for square obstacles at $12.7 \%$ blockage. At $6.3 \%$ blockage, however, $C_{D}$ increases with $s_{y} / D$ towards a maximum value and then begins to decrease, increasing again slightly close to the wall. The two shapes of obstacle show similar wake recovery in terms of the size of the region where the drag coefficient is negative. For circular cylinders this occurs for $s_{x} / D<2$ and $s_{y} / D<0.25$. For

(a)

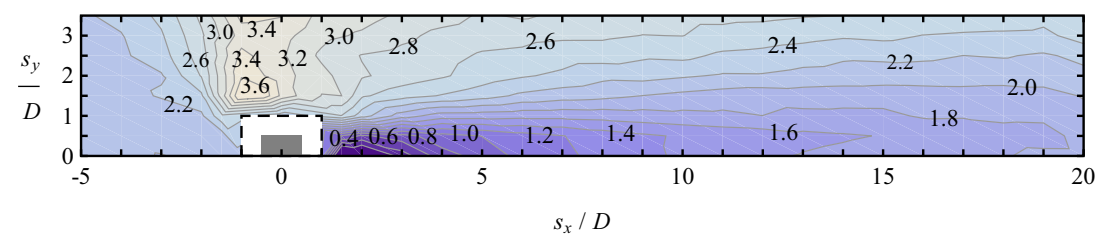

(b)

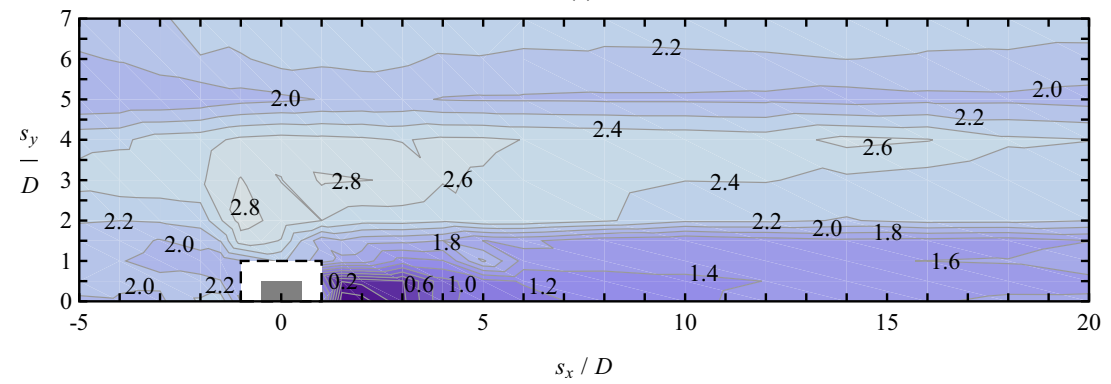

FIG. 12. Contours of drag coefficient on a second obstacle placed at different relative positions, with blockage ratios (a) $D / B=12.7 \%(\operatorname{Re}=16000)$ and (b) $D / B=6.3 \%(\operatorname{Re}=7500)$. 
square obstacles the drag is also negative for $s_{x} / D<2$ when they are in tandem, but the increments of $s_{y} / D$ were not small enough to measure negative drag for $s_{y} / D \neq 0$. Drag coefficients recover to an appreciable fraction of the isolated obstacle value at similar downstream distances, reaching $85 \%$ at $16 \mathrm{D}$ for circular cylinders in tandem and at $14 \mathrm{D}$ and $17 \mathrm{D}$ respectively for square obstacles at $6.3 \%$ and $12.7 \%$ blockage.

Blockage corrections were not applied to obstacle pairs because it has been demonstrated elsewhere that applying standard procedures with an effective blockage ratio (e.g., $D / B$ for tandem pairs and $2 D / B$ for side-by-side cylinders) is not sufficient. Utsunomiya et al. [32] proposed a modification to Maskell's theory [11], for tandem obstacles with rectangular cross-sections, where $\varepsilon$ is modified in proportion to a space factor which is a function of $s_{x} / D$. For staggered pairs one could anticipate a similarly modified $\varepsilon$ is a function of $s_{x} / D$ and $s_{y} / D$ and employ various values to collapse the data at the two blockage ratios to a single function, assuming that $\mathrm{Re}$ and $H / D$ effects are negligible. However, this is speculative and there is not enough data, with all other variables being equal, to verify the correction procedure is working accurately. In addition, for nearly side-by-side arrangements both the effective blockage ratio and $C_{D}$ are high compared to studies with isolated obstacles. Under these conditions, agreement between Maskell [11] and Ranga Raju and Singh's [13] correction methods breaks down, suggesting that at least one of the corrections is no longer valid even for a single obstacle. An investigation of blockage correction methods for staggered obstacle pairs is recommended for further study.

\section{CONCLUSIONS}

A comprehensive and careful set of experiments has been conducted to measure drag on pairs of square obstacles in subcritical open channel flow with parameters $\left(\operatorname{Re}, I_{T}\right)$ appropriate for many practical applications. The drag coefficient for a single square obstacle was found to be $C_{D}=2.11$, in agreement with earlier studies, suggesting that any increase due to blockage effects or the formation of surface waves was compensated for by the comparatively high $I_{T}$. It was also confirmed that $C_{D}$ is not strongly dependent on $\mathrm{Re}$ (unlike the drag on circular cylinders). The isolated obstacle $C_{D}$ was found to provide an estimate of the drag on an obstacle in a tandem pair (for $s_{x} \leqslant-3 D$ and $s_{x} \geqslant 4 D$ ) to within $10 \%$ accuracy if $U$ is replaced by $\bar{u}$ in the free stream, in the absence of the obstacle under consideration, where its horizontal center would be located $\left(x=s_{x}\right)$. Thus, for distances far enough away from the obstacle the change in streamwise speed is the primary reason for a change in drag, but when the obstacles are closer together the nature of the flow changes, giving a very different drag. This has important implications for estimating drag where there are a number of obstacles, as it suggests that to correctly predict the drag on downstream obstacles it is important to accurately model the temporally averaged streamwise velocity. If the same scaling of drag, based on the induced speed, also applies to side-by-side pairs then blockage effects are essentially just a subset of the flow speed alterations due to the presence of the first obstacle. A more general investigation which aims to explain drag changes, in staggered pairs or arrays, in terms of induced speed changes is recommended for further study. The root-mean-square drag coefficient was found to be a monotonically decreasing function of Re, based on hydraulic radius, and approaches 0.241 at high $\mathrm{Re}$ in agreement with previous studies and was independent of whether the flow depth is uniform across the obstacle.

For pairs of obstacles, the minimum $C_{D}$ occurred for a tandem arrangement where $C_{D}$ becomes negative for the downstream obstacle. The smallest values measured were for $s_{x}=1.5 D$, with $C_{D}=-0.16(D / B=6.3 \%)$ and $C_{D}=-0.40(D / B=12.7 \%)$. The maximum $C_{D}$ at $D / B=$ $12.7 \%$ is 3.82 , compared to only 2.85 at $D / B=6.3 \%$, and occurs when the obstacles are nearly side by side on the slightly upstream obstacle of a pair. The recovery of the wake and thus drag on an obstacle directly downstream is slow, with the drag on a downstream obstacle reaching $80 \%$ of the isolated value for $s_{x}=14 D(D / B=12.7 \%)$. Thus, laboratory and numerical models need to extend at least this far in the downstream direction to accurately capture the flow. The present study is restricted to angle of attack $\theta=0^{\circ}$, but it is clear that $\theta$ is an important parameter which 
can greatly affect $C_{D}$. As such, the influence of $\theta$ for both obstacles in a staggered pair, particularly at $12^{\circ}$ and $45^{\circ}$ where the minimum and maximum $C_{D}$ occur respectively for isolated obstacles, is recommended for further study, as this is important for practical applications such as wind induced airflow past nearby buildings.

The drag values found here can be used in a number of practical applications where it is necessary to estimate the forces on downstream structures or other bluff obstacles with similar shapes. The results can also be used to validate numerical models, where getting accurate results for the drag on a downstream obstacle (even for a square obstacle with well-defined separation points) is found to be challenging. In numerous applications (such as vegetation in streams or on flood plains and marine or riverine structures) obstacles are present in regular or random arrays, and the drag on arrays of square obstacles is the subject of our further study.

\section{ACKNOWLEDGMENT}

Francis H. Robertson was supported by a studentship from EPSRC (Grant Reference No. 1376075).

[1] D. J. Ball, P. K. Stansby, and N. Alliston, Modelling shallow water flow around pile groups, Proc. Inst. Civ. Eng. Water Marit. Energy 118, 226 (1996).

[2] S. C. Yen, K. C. San, and T. H. Chuang, Interactions of tandem square cylinders at low Reynolds numbers, Exp. Therm. Fluid Sci. 32, 927 (2008).

[3] S. C. Yen and C. W. Yang, Flow patterns and vortex shedding behavior behind a square cylinder, J. Wind Eng. Ind. Aerodyn. 99, 868 (2011).

[4] S. C. Yen and J. H. Liu, Wake flow behind two side-by-side square cylinders, Int. J. Heat Fluid Flow 32, 41 (2011).

[5] H. M. Nepf, Drag, turbulence, and diffusion in flow through emergent vegetation, Water Resour. Res. 35, 479 (1999).

[6] Y. Tanino and H. M. Nepf, Laboratory investigation of mean drag in a random array of rigid, emergent cylinders, J. Hydraul. Eng. 134, 34 (2008).

[7] N.-S Cheng and H. T. Nguyen, Hydraulic radius for evaluating resistance induced by simulated emergent vegetation in open-channel flows, J. Hydraul. Eng. 137, 995 (2011).

[8] R. Manning. On the flow of water in open channels and pipes, Trans. Inst. Civ. Eng. Ireland 20, 161 (1891).

[9] F. M. White, Viscous Fluid Flow 2nd ed. (McGraw-Hill, New York, 1991).

[10] J. Anthoine, D. Olivari, and D. Portugaels, Wind-tunnel blockage effect on drag coefficient of circular cylinders, Wind Struct. 12, 541 (2009).

[11] E. C. Maskell, A Theory of the Blockage Effects on Bluff Bodies and Stalled Wings in a Closed Wind Tunnel, Aeronautical Research Council Reports and Memoranda No. 3400 (Her Majesty's Stationery Office, London, 1965).

[12] H. B. Awbi, Wind-tunnel-wall constraint on two-dimensional rectangular section prisms, J. Wind Eng. Ind. Aerodyn. 3, 285 (1978).

[13] K. G. Ranga Raju, and V. Singh, Blockage effects on drag of sharp-edged bodies, J. Ind. Aerodyn. 1, 301 (1976).

[14] M. R. Raupach, Drag and drag partition on rough surfaces, Boundary-Layer Meteorol. 60, 375 (1992).

[15] X. Liu, M. Levitan, and D. Nikitopoulos, Wind tunnel tests for mean drag and lift coefficients on multiple circular cylinders arranged in-line, J. Wind Eng. Ind. Aerodyn. 96, 831 (2008).

[16] M. M. Zdravkovich and D. L. Pridden, Interference between 2 circular cylinders; series of unexpected discontinuities, J. Ind. Aerodyn. 2, 255 (1977).

[17] C. S. James, U. K. Goldbeck, A. Patini, and A. A. Jordanova, Influence of foliage on flow resistance of emergent vegetation, J. Hydraul. Res. 46, 536 (2008). 
[18] S. Wunder, B. Lehmann, and F. Nestmann, Determination of the drag coefficients of emergent and just submerged willows, Int. J. River Basin Manage. 9, 231 (2011).

[19] D. A. Lyn, S. Einav, W. Rodi, and J.-H. Park, A laser-Doppler velocimetry study of ensemble-averaged characteristics of the turbulent near wake of a square cylinder, J. Fluid Mech. 304, 285 (1995).

[20] C. Norberg, Flow around rectangular cylinders: pressure forces and wake frequencies, J. Wind Eng. Ind. Aerodyn. 49, 187 (1993).

[21] B. E. Lee, The effect of turbulence on the surface pressure field of a square prism, J. Fluid Mech. 69, 263 (1975).

[22] British Standards Institution, BS EN 1991-1-4, Eurocode 1: Actions on structures - General actions - Wind actions (British Standards Institution, London, 2005).

[23] H. Sakamoto, H. Haniu, and Y. Obata, Fluctuating forces acting on two square prisms in a tandem arrangement, J. Wind Eng. Ind. Aerodyn. 26, 85 (1987).

[24] M. K. Kim, D. K. Kim, S. H. Yoon, and D. H. Lee, Measurements of the flow fields around two square cylinders in tandem arrangement, J. Mech. Sci. Technol. 22, 397 (2008).

[25] W. Rodi, Comparison of LES and RANS calculations of the flow around bluff bodies, J. Wind Eng. Ind. Aerodyn. 69-71, 55 (1997).

[26] A. Sohankar and A. Etminan, Forced convection heat transfer from tandem square cylinders in cross flow at low Reynolds numbers, Intl J. Numer. Meth. Fluids 60, 773 (2009).

[27] A. Lankadasu and S. Vengadesan, Interference effect of two equal-sized square cylinders in tandem arrangement: with planar shear flow, Intl J. Numer. Methods Fluids 57, 1005 (2007).

[28] Nortek AS, Vectrino velocimeter user guide October 2004 Rev. c.

[29] F. H. Robertson, An experimental investigation of the drag on idealised rigid, emergent vegetation and other obstacles in turbulent free-surface flows, Ph.D. thesis, University of Manchester, UK, 2016.

[30] M. Escudier, Introduction to Engineering Fluid Mechanics (Oxford University Press, New York, 2017).

[31] E. Simiu and R. H. Scanlan, Wind Effects on Structures 3rd ed. (John Wiley \& Sons, New York, 1996).

[32] H. Utsunomiya, F. Nagao, Y. Ueno, and M. Noda, Basic study of blockage effects on bluff bodies, J. Wind Eng. Ind. Aerodyn., 49, 247 (1993). 